\title{
Digital autoradiography using CCD and CMOS imaging technology
}

\author{
J. Cabello, A. Bailey, I. Kitchen, A. Clark, J. Crooks, R. Halsall, M. Key-Charriere, S. Martin, M. \\ Prydderch, R. Turchetta and K. Wells
}

\begin{abstract}
CCD and CMOS imaging technologies can be applied to thin tissue Autoradiography as potential imaging alternatives to using conventional film. In this work, we compare two particular devices; a CCD operating in slow scan mode and a CMOS-based Active Pixel sensor, operating at near video rates. Both imaging sensors have been operated at room temperature with images produced from calibrated microscales and radiolabelled tissue samples. We also compare these digital imaging technologies with the use of conventional film. We show first comparative results obtained with ${ }^{14} \mathrm{C}$ calibrated microscales and ${ }^{35} \mathrm{~S}$ radiolabelled tissue sections. We also present first results of ${ }^{3} \mathrm{H}$ images produced under direct irradiation of a CCD sensor operating at room temperature. Compared to film, silicon-based imaging technologies exhibit enhanced sensitivity, dynamic range and linearity.
\end{abstract}

Index Terms-Digital Autoradiography, CCD, CMOS, APS, tissue imaging, high resolution.

\section{INTRODUCTION}

Autoradiography (AR) is a widely used technique in biology to map the two dimensional bio-distribution of radiolabelled molecules within thin $(\sim 10 \mu \mathrm{m})$ ex-vivo tissue sections. The aim of this technique is to qualitatively observe the relative distribution of the tracer, or, in many cases, to quantify specific areas of uptake (using simultaneous imaging of calibrated microscales). Traditionally, photographic film has been used as the imaging technology for AR. This exhibits excellent spatial resolution $(\sim$ few $\mu \mathrm{m})$ for low cost $(\sim \$ 3$ per sheet for standard film and $\sim \$ 50$ per sheet for ${ }^{3} \mathrm{H}$ imaging). But this technique presents a number of undesirable qualities, particularly with respect to image quantification. These include poor linearity, limited dynamic range $\left(\sim 10^{2}\right)$ and low sensitivity, producing long (typically $\sim$ days, $\sim$ months) exposure times. Alternative techniques based on digital imaging technology have been proposed to replace film. Whilst many authors have claimed that digital imaging technologies have superior sensitivity compared with film, we are not aware of any systematic study. In this work we attempt to make a first comparison between film and two exemplar imaging sensors.

Manuscript received November 17, 2006.

J. Cabello and K. Wells are with the Centre for Vision, Speech and Signal Processing, School of Electronics and Physical Sciences, University of Surrey, Guildford, Surrey, GU2 7XH, UK.

(email: j.cabello@ surrey.ac.uk)

A. Bailey and I. Kitchen are with the School of Biomedical and Molecular Sciences, University of Surrey, Guildford, Surrey, GU2 7XH UK.

A. Clark, J. Crooks, R. Halsall, M. Key-Charriere, S. Martin, M. Prydderch and R. Turchetta are with the Rutherford Appleton Laboratory, CCLRC, Chilton, OX11 0QX, UK.
Some of the more important techniques proposed as alternatives to film in AR include phosphor plates [1], [2], microchannel plates [3], [4] a Beta Camera [5] based on plate technology, multiwire proportional chamber systems [6] and solid state detectors [7], [8]. Our work presented here is focused in the latter area. Within solid state detectors several different technologies can be distinguished including CCD technology [8], CMOS technology [9] and hybrid detector technology [7]. In this work we examine two solid state alternatives to film: a commercial CCD sensor and a CMOS Monolithic Active Pixel sensor, both under direct irradiation from a radiolabelled sample. From an engineering perspective, CCD sensor technology has traditionally provided the "gold standard" in digital image performance in terms of high fill factor, quantum efficiency, small pixel size, dynamic range and low noise floor. However, CMOS imaging technology has made great strides in performance, and offers a potentially lower cost alternative additionally providing low power consumption, high level of integration, radiation hardness and high-speed operation.

The application of CCD technology for AR under cooled conditions has been clearly demonstrated previously [8]. Preliminary work at room temperature using CCDs under direct irradiation has also been reported [10]. In this new work we further examine the potential of these solid state imaging technologies operating at room temperature.

\section{MATERIALS AND METHODS}

The most common radioisotopes used in $\mathrm{AR}$ are ${ }^{32} \mathrm{P},{ }^{33} \mathrm{P}$, ${ }^{35} \mathrm{~S},{ }^{14} \mathrm{C}$ and ${ }^{3} \mathrm{H}$, shown in TABLE I.

TABLE I

COMMON RADIOISOTOPES USED IN AR

\begin{tabular}{cccc} 
Radioisotope & Average energy & Maximum energy & Half life \\
\hline${ }^{32} \mathrm{P}$ & $0.7 \mathrm{MeV}$ & $1.71 \mathrm{MeV}$ & 14.3 days \\
${ }^{33} \mathrm{P}$ & $76 \mathrm{keV}$ & $249 \mathrm{keV}$ & 25.3 days \\
${ }^{35} \mathrm{~S}$ & $48-53 \mathrm{keV}$ & $167 \mathrm{keV}$ & 87.4 days \\
${ }^{14} \mathrm{C}$ & $49 \mathrm{keV}$ & $156 \mathrm{keV}$ & 5730 years \\
${ }^{3} \mathrm{H}$ & $5.7 \mathrm{keV}$ & $18.6 \mathrm{keV}$ & 12.26 years \\
\hline
\end{tabular}

Tritium, or ${ }^{3} \mathrm{H}$, in particular, can label many sites on most biomolecules and provides the highest resolution due to its low energy (and hence low particle range, e.g. $\sim 0.2 \mu \mathrm{m}$ and $\sim 2.5 \mu \mathrm{m}$ for mean and maximum energy betas respectively in silicon) and therefore is one of the most common labels in molecular AR imaging studies. 


\section{A. Imaging System Description}

We have used, in our initial experiments, a CCD55-20 inverted mode sensor (e2v) [11] and a StarTracker active pixel sensor (APS) [9] to detect ${ }^{14} \mathrm{C}$ and ${ }^{35} \mathrm{~S}$. A back-illuminated CCD55-20 sensor has also been used to make the first demonstrated ${ }^{3} \mathrm{H}$ imaging at room temperature using a CCD under direct irradiation.

The CCD is a $770 \times 1152$ array of pixels on a $22.5 \mu \mathrm{m}$ pitch with a depletion region of $7 \mu \mathrm{m}$ and a sensitive field free region of $13 \mu \mathrm{m}$. The charge to voltage conversion is $3 \mu \mathrm{V} / \mathrm{electron}$ as quoted by the manufacturer [11]. The CCD has a 3-phase structure and is read out in full frame slow scan mode at a rate of $10 \mathrm{~s} /$ frame using correlated double sampling. The passivation layers, comprised of the electrodes layer and oxide layers, is $\sim 1.5 \mu \mathrm{m}$.

The CMOS StarTracker APS is a 525 x 525 array of pixels on a $25 \mu \mathrm{m}$ pitch with $5 \mu \mathrm{m}$ of overlying passivation layers and a depletion region of $4 \mu \mathrm{m}$. Each APS pixel has a structure made of 3 transistors (a reset MOSFET, a source follower MOSFET, which converts signal charge to voltage, and a row-select MOSFET) and four n-well/p-substrate photodiodes placed on a $12.5 \mu \mathrm{m}$ pitch. This architecture optimizes the charge collection having, at the same time, low node capacitance $(\sim 18 \mathrm{fF})$ in order to give better charge to voltage conversion $(\sim 8.9$ $\mu \mathrm{V} /$ electron). The readout is column-parallel with an adjustable gain amplifier and a 10-bit ramp ADC per column that reads out at near video rates (10 frames $\left.\mathrm{s}^{-1}\right)$.

In order to correct for dark current and inter-pixel nonuniformities, every image acquired on each system is processed using Matlab-based software. For the case of the CCD, first a post-acquisition dynamic Fixed Pattern Noise (FPN) correction is applied to every image. For the case of the APS, a set of blank frames is previously acquired and used to compute the mean of each pixel, referred to as a static FPN correction. The mean of each blank pixel is subtracted from each observed pixel intensity in the acquired image. After this first step, the subsequent processing is the same for both sensors. A statistical threshold is then applied to each pixel defined as the mean (in absence of signal) of each pixel plus $k$ standard deviations of each pixel, using prior knowledge of the mean and variance of each pixel acquired under dark level conditions. The resulting binary images are then labelled using 8-connectivity analysis and the size and intensity of each event cluster is saved. To obtain a useful composite image the thresholded images, described above, were accumulated and summed.

The result of this correction is shown in the fig. 1 . Note the scale of each image.

In the case of the CMOS sensor the images were acquired with an integration time of 100 msecs. A static blank background was subtracted from each acquired image. Posterior thresholding then applied to every corrected image is sufficient to obtain a largely "clean" composite image. See fig. 2.

\section{B. Calibration}

In order to compare sensor performance it was necessary to calibrate the energy response of both imaging systems, so that

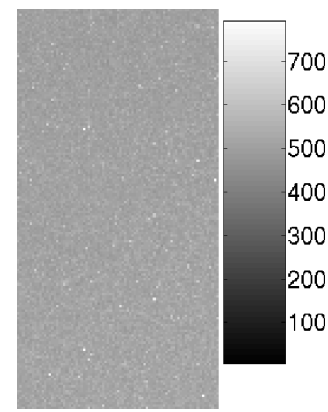

(a) Raw image

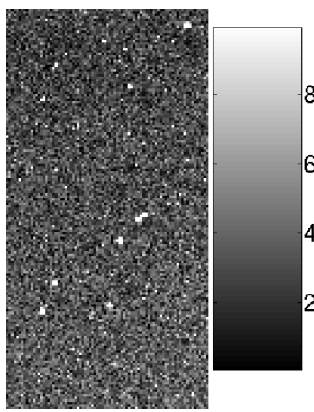

(b) Corrected image

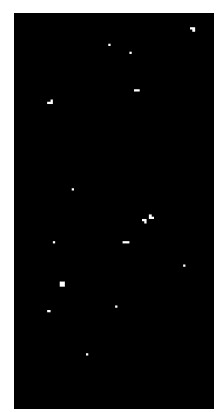

(c) Thresholded image
Fig. 1. Raw, corrected and thresholded images of the front illuminated CCD sensor, showing the stages used to correct for pattern noise.

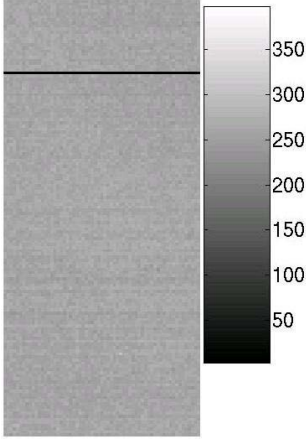

(a) Raw image

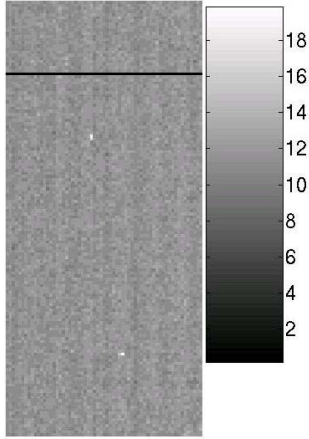

(b) Corrected image

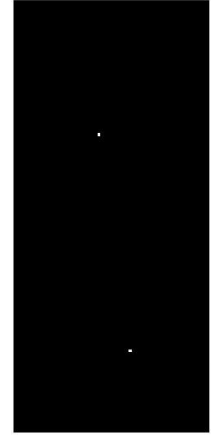

(c) Thresholded image
Fig. 2. Raw, corrected and thresholded images of the front illuminated CMOS sensor.

a comparative event threshold in calibrated $\mathrm{keV}$ units could be used. The CCD sensor was exposed to a point source of ${ }^{241} \mathrm{Am}$ (activity of $41 \mathrm{kBq}$ ) situated around $30 \mathrm{~mm}$ from the CCD with a layer of insulating tape to absorb the associated $\alpha$ particles.

Peaks corresponding to the ${ }^{241} \mathrm{Am}$ photon energies of 11.9 $\mathrm{keV}, 13.9 \mathrm{keV}, 17.5 \mathrm{keV}, 22 \mathrm{keV}$ and $26 \mathrm{keV}$ were observed.

Exposing the $\mathrm{CCD}$ sensor to a ${ }^{14} \mathrm{C}$ source also provided a reference mean energy of $49 \mathrm{keV}$ from the associated beta energy deposited spectrum. From these data, a fitted line is shown in fig. 3 with a slope of $0.36 \mathrm{keV} / \mathrm{ADC}$ unit, from which $1 \mathrm{keV}$ corresponds to 9.72 ADC units. Assuming $3.6 \mathrm{eV}$ is required to liberate 1 electron in silicon, then this yields a slope of 100 electrons/ADC unit.

Expressing the threshold applied to the corrected images acquired with the CCD sensor in $\mathrm{keV}$, a mean threshold of $6.7 \mathrm{keV}$ is obtained with an approximately Gaussian variation of width $\sigma=1.9 \mathrm{keV}$. This width represents the variation in effective pixel threshold used, due to inter-pixel variations in performance.

In the case of the APS sensor, it was not possible to calibrate with a beta or x-ray source because of the thin epi layer of the sensor $(4 \mu \mathrm{m})$. This therefore calibration was undertaken using the Photon Transfer Technique. Using corresponding values taken from [12] the resulting calibration plot is shown in fig. 4. 


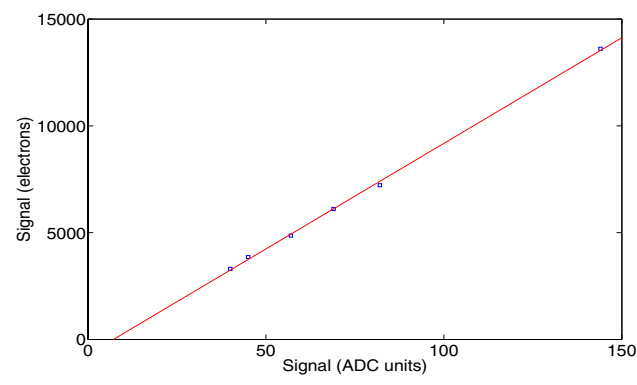

Fig. 3. Energy calibration curve for $\mathrm{CCD}$ sensor. $\mathrm{X}$ axis represents the signal in $\mathrm{ADC}$ units and $\mathrm{Y}$ axis represents the signal in electrons.

Expressing the threshold applied to the corrected images in $\mathrm{keV}$, in the case of the APS, a mean threshold of $8.5 \mathrm{keV}$ is obtained with an approximately Gaussian variation of width $\sigma$ $<1 \mathrm{keV}$.

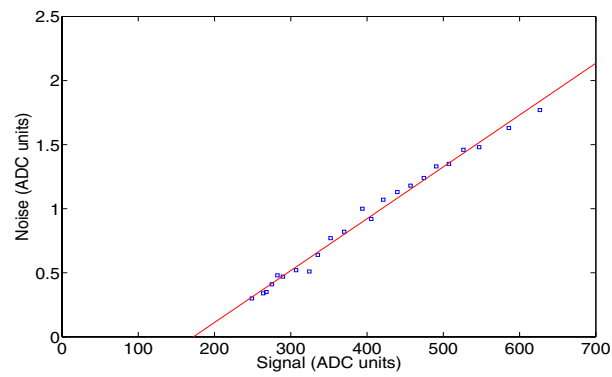

Fig. 4. PTC curve for CMOS sensor. $\mathrm{X}$ axis represents the signal (mean) in $\mathrm{ADC}$ units and $\mathrm{Y}$ axis represents the noise (variance) in ADC units.

\section{Image Acquisition}

Preliminary results obtained using calibrated microscales of ${ }^{14} \mathrm{C}$ and ${ }^{3} \mathrm{H}$ are presented below. These microscales consist of known amounts of radioactivity homogeneously distributed in a plastic tissue equivalent polymer of 5-10 $\mu$ m thickness. Each microscale has 8 cells or varying activity; for ${ }^{3} \mathrm{H}$ the range is $0.11-4.04 \mathrm{kBq} / \mathrm{mg}$ and for ${ }^{14} \mathrm{C}$ is $1.11-31.89 \mathrm{kBq} / \mathrm{g}$ [13]. The experiments were set up placing each sensor in a light proof box and placing the microscale in direct contact with the surface of the sensor. The exposure time of the experiments with ${ }^{14} \mathrm{C}$ for the CCD (fig. 5) and the APS (fig. 6) was 1 hour and 40 minutes. For ${ }^{3} \mathrm{H}$ experiments using the $\mathrm{CCD}$, the exposure time was 11 hours with the microscales (fig. 14), and 16 hours for the tissue specimen with ${ }^{35} \mathrm{~S}$ (figs. 12 and 13).

\section{Preliminary Results \& Discussion}

\section{A. Sensitivity}

In fig. 5 we present a composite image of calibrated ${ }^{14} \mathrm{C}$ microscales using the CCD sensor. Fig. 6 demonstrates the corresponding image obtained from the APS sensor with ${ }^{14} \mathrm{C}$, representing the first use of this particular APS imaging device for AR. Computing the number of events per band and dividing by the activity of each band, the sensitivity for each band

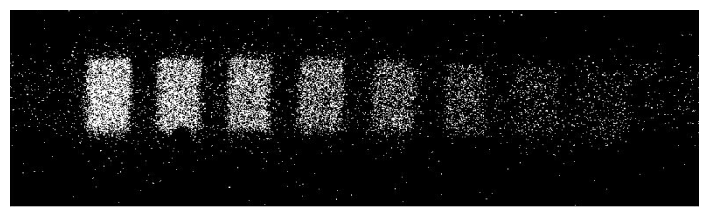

Fig. 5. Composite image with ${ }^{14} \mathrm{C}$ obtained with the CCD sensor after 100 minutes exposure

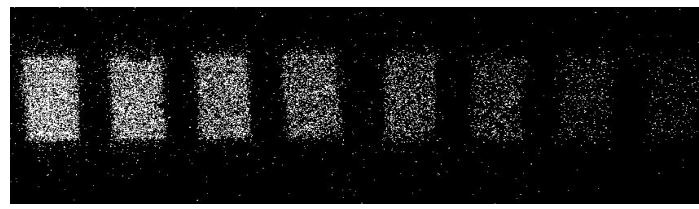

Fig. 6. Composite image with ${ }^{14} \mathrm{C}$ obtained with the CMOS sensor after 100 minutes exposure

is calculated. The mean of the sensitivity of each band is 233 counts $/ \mathrm{kBq} / \mathrm{g}$ for the CCD and 175 counts $/ \mathrm{kBq} / \mathrm{g}$ for the APS (1.33 times lower). Although both images appear visually similar this demonstrates the lower sensitivity of the CMOS sensor which is to be expected due to the higher fill factor of the CCD technology and the thinner epi layer of this APS sensor. This is also shown in fig. 8 that shows the number of detected events per $\mathrm{mm}^{2}$ in each band. This plot shows that the slope of the CMOS sensor ( 36.2 counts $/ \mathrm{kBq} / \mathrm{g} \mathrm{mm}^{2}$ ) is lower than that of the CCD sensor (43.3 counts / $\mathrm{kBq} / \mathrm{g} \mathrm{mm}^{2}$ ) meaning, again, that the sensitivity of the CCD sensor is higher than that of the APS.

From the ROI analysis described in Section I.A. the density of events in the background for the CCD was estimated at 20 events $/ \mathrm{mm}^{2}$ and for the APS at 9 events $/ \mathrm{mm}^{2}$ (2.22 times lower). This shows us that the APS sensor exhibits lower sensitivity but also lower background pixel noise than the CCD.

For comparison, we have also exposed a Biomax MR film to the same calibrated source for 100 minutes. The resulting image is shown in fig. 7. Clearly, the level of contrast against background fogging makes image quantification impossible at such a short exposure time.

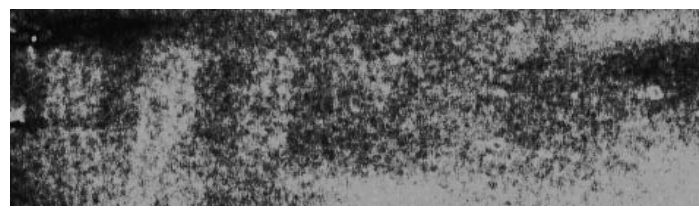

Fig. 7. Exemplar image produced using ${ }^{14} \mathrm{C}$ microscale after exposure to Biomax MR film for 100 minutes.

ROI analysis has been undertaken demonstrating excellent linear response for both sensors. In fig. 8 we present the number of detected events per $\mathrm{mm}^{2}$ for the CCD sensor (squares) and the CMOS sensor (crosses) as a function of source activity.

\section{B. Energy Spectra}

In fig. 9 we show the deposited energy spectrum of ${ }^{14} \mathrm{C}$ obtained with the CCD and the APS sensors. These spectra 


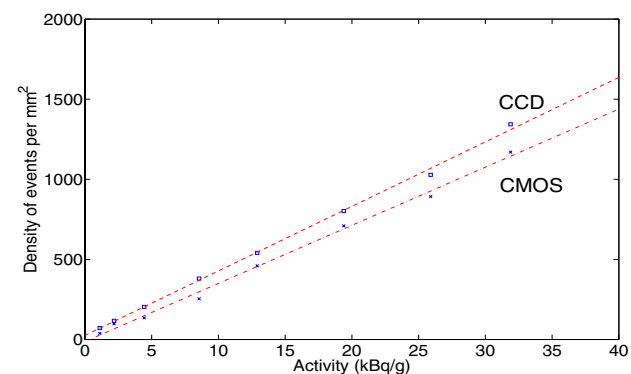

Fig. 8. Number of events $/ \mathrm{mm}^{2}$ for each band of the microscale of ${ }^{14} \mathrm{C}$ taken with the CCD sensor (squares) and the CMOS sensor (crosses).

were obtained computing the histogram of the intensity of every event from the data mentioned in the subsection I.A.

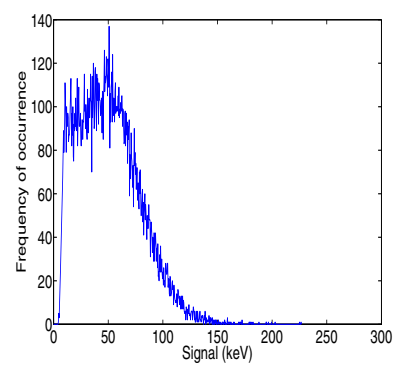

(a)

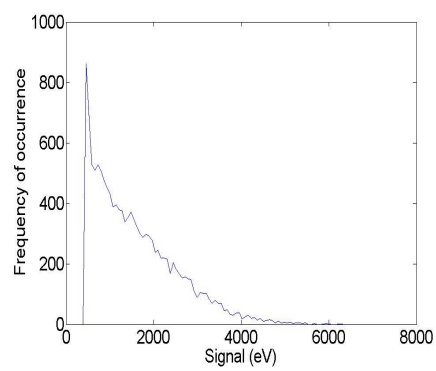

(b)
Fig. 9. Deposited energy spectrum of ${ }^{14} \mathrm{C}$ obtained with the CCD (fig. 9(a)) and the CMOS (fig. 9(b)) sensor. Note that in the case of 9(a), below $50 \mathrm{keV}$ the pattern noise correction has difficulties in correctly identify genuine events from the background pattern noise artifacts. 9(b) compared to 9(a) suggests the the CMOS sensor only partially samples the incident energy.

Note that the spectrum obtained with the CCD is similar to the expected continuous spectrum of ${ }^{14} \mathrm{C}$, in contrast to that exhibited by the CMOS APS sensor. This is attributed to the thickness of the epitaxial layer: in the CCD this is $20 \mu \mathrm{m}$ whereas in the CMOS sensor, it is $4 \mu \mathrm{m}$. The range of the beta particles from ${ }^{14} \mathrm{C}$ in silicon is $16 \mu \mathrm{m}$ for the mean energy and $121 \mu \mathrm{m}$ for the maximum energy. This means that in the thin epitaxial layer of the CMOS device, we believe that the sensor behaves as a thin $d E / d x$ detector, partially sampling the particle energy, compared to the CCD, where there is a relatively high chance of observing the full beta energy deposition.

\section{Size of Events}

In fig. 10 we show the histograms of different cluster sizes of the detected events from ${ }^{14} \mathrm{C}$. This was obtained computing the histograms of the size of every event from the arrays mentioned in the subsection I.A. This shows that, in both sensors, most of the detected events are 1-2 pixels in size and those events over 4-5 pixels are not normally detected. Note the CCD, with its thicker sensitive region, produces relatively more of the larger $(>2$ pixels) events.

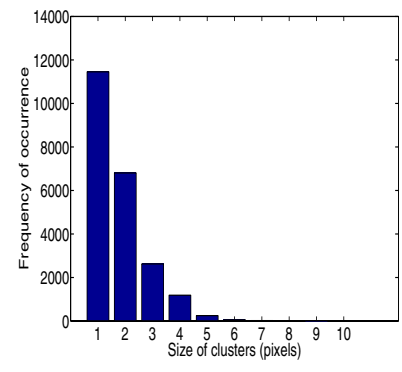

(a)

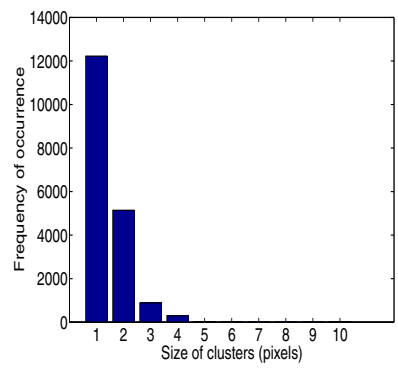

(b)
Fig. 10. Size of events with ${ }^{14} \mathrm{C}$ obtained with the CCD (fig. 10(a)) and with the CMOS (fig. 10(b)).

\section{Tissue Imaging}

To examine the imaging system performance under realistic conditions various tissue sections were prepared. Several mouse tissue sections were preincubated with $1 \mathrm{mM} \mathrm{GDP}^{1}$ and then incubated for 2 hours with ${ }^{35} \mathrm{SGTP} \gamma \mathrm{S}^{2}(0.04 \mathrm{nM})$ and $1 \mathrm{mM}$ GDP [14]. These sections shown are from the level of the Caudate (Bregma 1.00mm) (fig. $11 \mathrm{left}$ ) and from the level of the Hippocampus (Bregma $2.56 \mathrm{~mm}$ ) (fig. 11 right) bonded with ${ }^{35} \mathrm{SGTP} \gamma \mathrm{S}$. The physical size of these sections was measured as $10.4 \mathrm{~mm} \times 6.8 \mathrm{~mm}$. After this preparation the sections were placed within a film cassette, in direct contact with film, for $\sim 4$ days for ${ }^{35} \mathrm{~S}$.

To demonstrate the comparative biological imaging potential of CCD and APS technology compared to film, exemplar images produced using direct irradiation of the same radiolabelled biological mouse samples are presented in figs. 11, 12 and 13 . This shows a comparison of images taken with film (fig. 11) for four days (5760 minutes, the usual exposure time used in our laboratory for these studies) and with the CCD (fig. 12) and with the APS sensor (fig. 13) exposed for $10^{3}$ minutes ( $~ 6$ times shorter). These images were then smoothed with a $3 \times 3$ block filter applied twice.

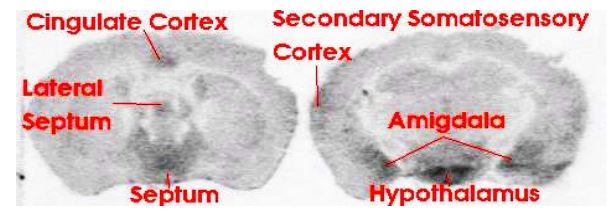

Fig. 11. Film-based AR images from coronal mouse sections, from the level of the Caudate (Bregma 1.00mm) (left) and from the level of the Hippocampus (Bregma $2.56 \mathrm{~mm}$ ) (right), bonded with ${ }^{35} \mathrm{SGTP} \gamma \mathrm{S}$ exposed to film for four days

\section{E. ${ }^{3}$ H Imaging}

As mentioned above, the most common radioisotope in AR is ${ }^{3} \mathrm{H}$. The energy of this isotope is so low that in a frontilluminated device, the beta particles would be stopped in the overlying passivation layers. Therefore a back-thinned CCD5520 device was substituted for ${ }^{3} \mathrm{H}$ imaging to demonstrate the

\footnotetext{
${ }^{1}$ Guanosine 5'-Diphosphate binding

${ }^{2}$ Gamma-thio-triphosphate gamma $\mathrm{S}$ binding bonded with ${ }^{35} \mathrm{~S}$
} 


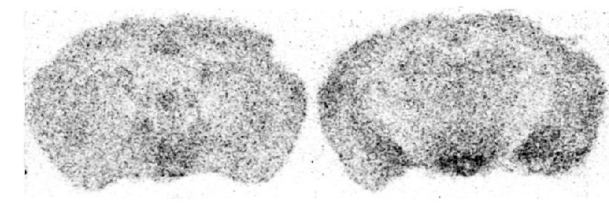

Fig. 12. Coronal mouse sections, from the level of the Caudate (Bregma $1.00 \mathrm{~mm}$ ) (left) and from the level of the Hippocampus (Bregma $2.56 \mathrm{~mm}$ ) (right), bonded with ${ }^{35} \mathrm{SGTP} \gamma \mathrm{S}$ exposed to CCD for 16 hours

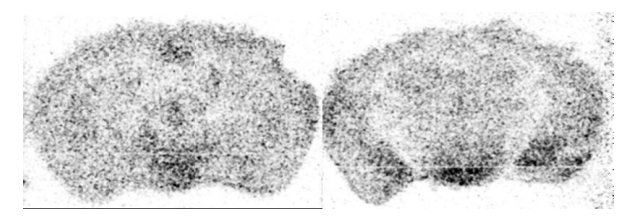

Fig. 13. Coronal mouse sections, from the level of the Caudate (Bregma $1.00 \mathrm{~mm}$ ) (left) and from the level of the Hippocampus (Bregma $2.56 \mathrm{~mm}$ ) (right), bonded with ${ }^{35} \mathrm{SGTP} \gamma \mathrm{S}$ exposed to CMOS for 16 hours

potential of pixelated detectors operating at room temperature and using direct irradiation.

Despite the sub-optimized read-out system used, in fig. 14 we show the first ${ }^{3} \mathrm{H}$ image obtained with a $\mathrm{CCD}$ at room temperature under direct irradiation.

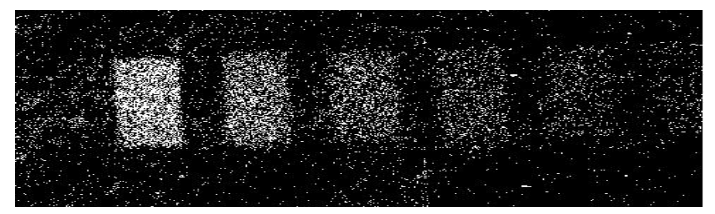

Fig. 14. Composite image with ${ }^{3} \mathrm{H}$ obtained with the CCD after 11 hours.

Undertaking the same ROI analysis as undertaken with ${ }^{14} \mathrm{C}$, in figs. 15 and 16 we show the energy spectrum of ${ }^{3} \mathrm{H}$ and the number of events per $\mathrm{mm}^{2}$ of this radioisotope with the back-illuminated CCD. From fig. 14, the sensitivity measured is 0.56 counts $/ \mathrm{kBq} / \mathrm{g}$ and the density of events in the background is 64 events $/ \mathrm{mm}^{2}$. From fig. 16 the slope measured is 0.15

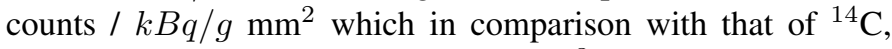
shows an expected lower sensitivity to ${ }^{3} \mathrm{H}$ beta-particles. The high number of events in the background is explained by the sub-optimized read-out electronics and Fixed Pattern Noise correction. Using the same calibration procedure as undertaken for the front-illuminated CCD sensor, the back-thinned device was found to use a threshold of $3.46 \mathrm{keV}$ with an inter-pixel variation of $0.3 \mathrm{keV}$.

For comparison with film, in fig. 17, we present an image obtained from digitizing a sheet of tritiated hypersensitive hyperfilm exposed to a ${ }^{3} \mathrm{H}$ microscales for two weeks.

To make a comparative assessment against using conventional film we repeated this experiment for exposure periods of 100 minutes, one, two and four weeks, and applied a similar ROI analysis as described previously. To compare the sensitivity we initially exposed a microscale of ${ }^{3} \mathrm{H}$ to a sheet of tritiated hypersensitive hyperfilm for 11 hours, the same length of time used with the CCD sensor. The result was a completely blank

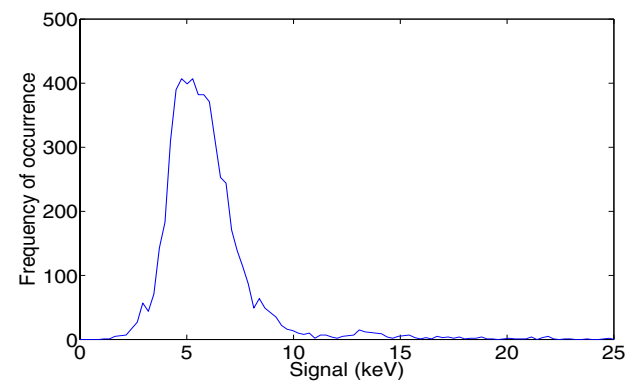

Fig. 15. Deposited energy spectrum of ${ }^{3} \mathrm{H}$ obtained with a back-thinned CCD.

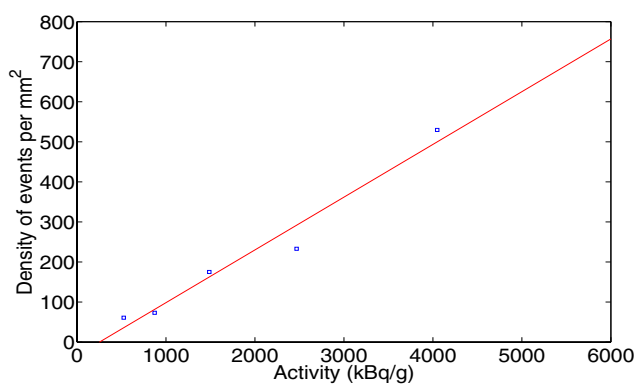

Fig. 16. Number of events $/ \mathrm{mm}^{2}$ for each band of the microscale of ${ }^{3} \mathrm{H}$ taken with the CCD sensor.

image discarding the typical background fogging. As can be seen in fig. 18 the resulting data can not be fitted with a straight line. This demonstrates the expected non linear response of film imaging compared to that of the digital devices. Note that for the two weeks exposure, the weakest band from the calibrated microscales is not detected and for the experiment, taken for one week exposure, the two weakest bands are not detected.

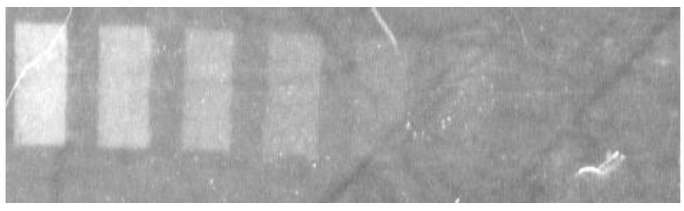

Fig. 17. Exemplar image produced using ${ }^{3} \mathrm{H}$ microscale after exposure to tritiated hypersensitive hyperfilm for two weeks.

\section{CONCLUSIONS}

We have demonstrated thus far that it is possible to use CCD and APS sensors operating at room temperature for AR, reducing exposure, thus improving sensitivity, and linearity, easing image quantification, and reducing exposure time compared to using traditional film. We have in addition demonstrated that the CMOS APS sensor shows lower sensitivity but it also presents a lower background noise floor, compared to the slow-scan CCD device. This makes this technology a serious choice to bare in mind for future digital AR.

We have also demonstrated that it is possible to detect ${ }^{3} \mathrm{H}$ radiation with a backthinned $\mathrm{CCD}$ operating at room temperature. 


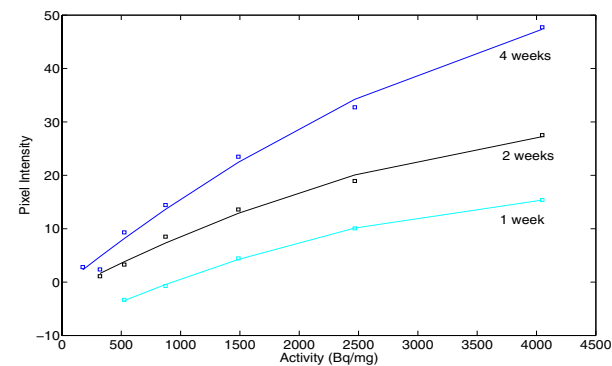

Fig. 18. Ratio of the mean value of each band and the mean value of the background. Experiment undertaken with ${ }^{3} \mathrm{H}$ exposed to film for one(cyan), two(black) and four weeks(blue).

\section{ACKNOWLEDGMENT}

The StarTracker APS sensor was originally designed and first manufactured under Natural Environment Research Council (NERC) funding. The work reported here was undertaken as part of the $\mathrm{MI}^{3}$ Basic Technology programme, funded by the Engineering and Physical Sciences Research Council. The CCD component of this work was funded by a University of Surrey Proof of Concept Award.

\section{REFERENCES}

[1] R. F. Johnston, S. C. Pickett, and D. L. Barker, "Autoradiography using storage phosphor technology," Electrophoresis, vol. 11, pp. 355-360, 1990.

[2] W. L. Reichert, J. E. Stein, B. French, P. Goodwin, and U. Varanasi, "Storage phosphor imaging technique for detection and quantitation of DNA adducts measured by the 32P-postlabeling assay," Carcinogenesis, vol. 13, pp. 1475-1479, 1992.

[3] J. E. Lees, A. Murray, A. C. Perkins, and G. W. Fraser, "Autoradiography of High-Energy radionuclides using a microchannel plate detector," IEEE Transactions of Nuclear Science, vol. 49, pp. 153-155, 2002.

[4] J. E. Lees, J. F. Pearson, G. W. Fraser, J. M. Hales, and P. G. Richards, "An MCP-based system for beta autoradiography," IEEE Transactions of Nuclear Science, vol. 46, pp. 636-638, 1999.

[5] K. Ljunggren and S. E. Strand, "Beta camera for static and dynamic imaging of charged-particle emitting radionuclides in biologic samples," Journal of Nuclear Medicine, vol. 31, pp. 2058-2063, 1990.

[6] Y. Petegnief, A. Petiet, M. C. Peker, F. Bonnin, A. Meulemans, and D. Guludec, "Quantitative autoradiography using a radioimager based on a multiwire proportional chamber," Physics in Medicine and Biology, vol. 43, pp. 3629-3638, 1998.

[7] G. Mettivier, M. C. Montesi, and P. Russo, "Tritium digital autoradiography with a Medipix2 hybrid silicon pixel detector," Nuclear Instruments and Methods in Physics Research, vol. 516, 2004.

[8] R. J. Ott, J. MacDonald, and K. Wells, "The performance of a CCD digital autoradiography imaging system," Physics in Medicine and Biology, vol. 45, pp. 2011-2027, 2000.

[9] M. L. Prydderch, N. J. Waltham, R. Turchetta, M. J. French, R. Holt, A. Marshall, D. Burt, B. R., P. Pool, E. C., and H. Mapson-Menard, "A 512 x 512 CMOS Monolithic Active Pixel sensor with integrated ADCs for space science," Nuclear Instruments and Methods in Physics Research, vol. 512, pp. 358-367, 2003.

[10] E. Kokkinou, K. Wells, M. Petrou, and A. Bailey, "Digital autoradiography imaging using direct irradiation of a CCD between 278-309 K," IEEE Transactions of Nuclear Science, vol. 50, pp. 1702-1707, 2003.

[11] http://e2v.com/, E2V Technologies.

[12] K. Arvanitis, S. Bohndiek, G. Segneri, C. Venanzi, G. Royle, A. Clark, J. Crooks, R. Halsall, M. Key-Charriere, S. Martin, M. Prydderch, R. Turchetta, and R. Speller, "A novel active pixel sensor with on-pixel analog-to-digital converter for mammography," To appear in Conference Proceedings of IEEE NSS MIC. Medical Imaging, 2006.

[13] G. Healthcare UK Limited, Pollards Wood, Nightingales Lane, Chalfont St.Giles, Bucks, UK, and HP8 4SP.
[14] A. Bailey, H. M. B. Lesscher, M. Kelly, C. Ledent, L. Davis, S. M. O. Hourani, and K. I, "Enhanced morphine withdrawal and $\mu$-opioid receptor G-protein coupling in $\mathrm{A}_{2} \mathrm{~A}$ adenosine receptor knockout mice," Journal of Neurochemistry, vol. 88, pp. 827-834, 2004. 